\title{
The Excitement Theory on Human Behaviour
}

\author{
Dr. Asad Reza \\ (Patna Medical College, India)
}

\begin{abstract}
Human emotions are a complex manifestation of natural phenomena. However, these emotions to some extent can be mathematically expressed in form of simple equations. We have observed games and events and the reaction of the crowds to a particular situation. We find some events interesting and some dull. This subjective feeling has been mathematically expressed in form of an equation and termed as 'Excitement'. A numerical factor is obtained, the magnitude of which gives the degree of excitement.
\end{abstract}

Key-words: Biased, Depression, Event, Excitement, Impulse, Un-biased.

\section{The Excitement Theory}

The beauty of nature is that it manifests everywhere in the form of mathematical equations having simple base. In the physical world, we can see that a diverse and complex natural phenomena occurs. And we have tried to simplify the diverse phenomena in terms of physical concepts and laws, for the most complex natural phenomena can be explained in the simplest law and equation. Human behaviour is just the reflection of the material world . By analogy, human nature can be explained in terms of laws governing the physical world. It was through this analogy and symmetry in nature that led $d e$ Broglie to propose his theory on matter waves. If in nature, everything is so symmetrical that concept of matter could be explained in terms of energy, then surely human behaviour and laws governing the physical world ought to have some coherence.

We have both played and observed games. We find some matches exciting whereas some are dull and boring. We often find people suffering from heat-attacks after an interesting event or people committing suicide after failing. This paper is chiefly concentrated on describing the human emotions in mathematical equations and applications of the equations on related population groups. And as this is a subjective theory, and like all subjective theories the onus lies on the author to convince the readers, so I leave it to my readers to trust your intuition after you have assessed the contents. A successful theory will not only explain the present circumstances but will appeal to the wide majority, be scientific and stand the test of time.

\section{Introduction}

Suppose two terms A and B are playing a match. For an unbiased observer (who knows the game), the match will be interesting when both teams have equal chances of winning. The match will be uninteresting if one of the team is stronger and has greater chance of winning.

Mathematically 'excitement' is defined as "the interest generated in an activity". An interesting activity full of suspense will be more exciting than a one which lacks suspense. For example, a match played by two strong teams will be more exciting when they have equal chances of winning as the outcome cannot be easily proposed.

Excitement may be biased or unbiased. Unbiased excitement is when a natural observer watches a match without supporting any team or player. Whereas the excitement becomes biased when the observer supports one of the participants.

\section{Unbiased Excitement}

Suppose an unbiased observer watches a match between two teams A and B, whose probability of winning the match is ' $x$ ' and ' $y$ ' respectively. He will find the match more interesting when ' $x$ ' and ' $y$ ' approach each other. As the values of ' $x$ ' and ' $y$ ' differ in magnitude, the match will be less interesting. If we plot a graph correlating probability of occurrence of event with excitement, we get the following curve: 


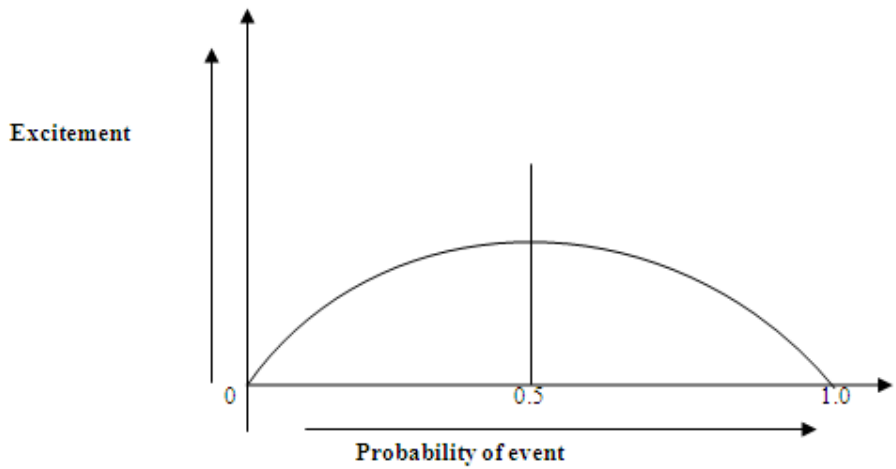

From the graph we can observe that there is no excitement when it is fully certain whether a team is going to win or lose.

To understand this situation consider throwing a die. If we take an event as say, a number less than seven. There will be no excitement in the event, because we know for sure that on throwing a die, a number less than seven will come.

Consider another situation as - an odd number on throwing a die. The event becomes interesting as there is a chance of both an odd number and even number appearing on the face of die.

Graphically, several models can be developed for the above observations, but it can be observed that excitement best follows a sine curve with probability of the event. Thus the following theorem can be proposed based on these observations.

Theorem I : The excitement for an event is directly proportional to the sine of pi $(\pi)$ times the probability of the event.

Mathematically, we can write it as :

$$
\begin{aligned}
& \text { Excitement }(\mathrm{E}) \propto \sin (\mathrm{p} \pi) \\
& \mathrm{E}=\mathrm{K} \sin (\mathrm{p} \pi)
\end{aligned}
$$

Where ' $\mathrm{p}$ ' is the probability of occurrence of the event and $\mathrm{K}$ is a constant.

$\mathrm{K}$ may vary from game to game and event to event. But considering the intrinsic emotional state in man, we assume $\mathrm{K}=1$ for all situations in an unbiased observer.

Thus,

$$
\mathrm{E}=\sin (\mathrm{p} \pi)
$$

The equation obtained is for a single or individual element associated with the event.

Now, when there are ' $n$ ' participants (each having their own identity) for an event and probability of event associated with each of them be $p_{1}, p_{2}, \ldots \ldots \ldots \ldots p_{n}$.

Theorem II : The excitement for an event with 'n' number of participants(each having their individual identity) will be the sum of excitement for each of the individual elements.

Mathematically,

$$
\begin{aligned}
& E_{1}=\sin \left(p_{1} \pi\right), E_{2}=\sin \left(p_{2} \pi\right), \ldots \ldots \ldots \ldots . E_{n}=\sin \left(p_{n} \pi\right) \\
& E=E_{1}+E_{2}+\ldots \ldots \ldots \ldots \ldots \ldots+E_{n} \\
& E=\sin \left(p_{1} \pi\right)+\sin \left(p_{2} \pi\right)+\ldots \ldots \ldots \ldots \ldots \ldots+\sin \left(p_{n} \pi\right)
\end{aligned}
$$

Now, let's define some terms related with excitement.

3.1. Excitement in an Event $\left(\mathbf{E}_{\mathbf{i}}\right)$ : It is defined as the degree of interest in an event which is about to occur and hasn't occurred yet.

If there are ' $n$ ' participants (individual identity), participating in an event and probability of event associated with each of them respectively be $\mathrm{p}_{1}, \mathrm{p}_{2}, \ldots \ldots \ldots \ldots \mathrm{p}_{\mathrm{n}}$

Then, the Excitement in the Event will be,

$$
E_{i}=\sin \left(p_{1} \pi\right)+\sin \left(p_{2} \pi\right)+\ldots \ldots \ldots \ldots \ldots \ldots+\sin \left(p_{n} \pi\right)
$$

Note :- The theorem explains why tournaments in which large number of teams(participants) participate are interesting than simply two team matches. It's the reason why World Cup events and Olympic games are more exciting.

The basis lies in the fact, that a large number of teams participate and due to the process of selection, each team has an overall average probability of winning. 
3.2. Excitement of an Event $\left(\mathbf{E}_{\mathbf{0}}\right)$ : It is defined as the interest generated after an event has occurred.

Theorem III : The excitement of an event that has occurred is directly proportional to the excitement in the event and inversely proportional to the probability of occurrence of that event.

Mathematically, it can be written as :

$$
\begin{array}{ll}
\mathrm{E}_{\mathrm{o}} \propto \mathrm{E}_{\mathrm{i}} & \text { (i) } \\
\text { And } \quad \mathrm{E}_{\mathrm{o}} \propto \frac{1}{\mathrm{P}} &
\end{array}
$$

Therefore,

$$
\begin{aligned}
& \mathrm{E}_{\mathrm{o}} \propto \frac{\mathrm{Ei}}{\mathrm{p}} \\
& \mathrm{E}_{\mathrm{o}}=\mathrm{K}_{\mathrm{o}} \mathrm{E}_{\mathrm{i}} / \mathrm{p}, \quad \text { [where } \mathrm{K}_{\mathrm{o}} \text { is a constant]. }
\end{aligned}
$$

Note :- This theorem explains why a match is more interesting when a weak team defeats a strong team. The occurrence of supernatural or unnatural events are always exciting due to their low probability of occurrence.

$\mathrm{K}_{\mathrm{o}}$ may vary with different events. But the intrinsic emotion in man for an unbiased observer is constant. So assuming $\mathrm{K}_{\mathrm{o}}=1$ for all situations in an unbiased observer :

$$
\mathbf{E}_{\mathbf{o}}=\mathbf{E}_{\mathrm{i}} / \mathbf{p}
$$

3.3. Excitement Impulse (E.I.) : It is the excitement generated after an event's occurrence due to unexpectancy of the occurrence of the event.

Theorem IV : The Excitement Impulse is the product of excitement of an event and probability of not expecting the events occurrence.

Suppose, the excitement of event be $\mathrm{E}_{\mathrm{o}}$ and the probability of occurrence of the event be ' $\mathrm{P}$ '.

Then,

$$
\text { E.I. }=\mathbf{E}_{0} \mathbf{x}[(\mathbf{1 - P})-\mathbf{P}(\text { Tie })]
$$

Where (1-P) is un-expectancy of the event's occurrence. Generally, for an unbiased observer, there is no excitement when a match ends in a draw.

\section{Biased Excitement}

This is practical and in general related to any arbitrary observer that we see. The bias arises out of past experience and is proportional to the time or money invested in the event and/or its history. Patriotism, nepotism and miscellaneous relationship with the participants (in case the observer is also the participant) makes an observer biased.

Suppose two strong teams play a match. They have equal chance of winning. It will be difficult to predict which team will win the match. So the event has high excitement in it.

Now if two weak teams are playing a match, then both the teams will have equal chance of winning. But practically, the match isn't as interesting as that was when two strong teams were playing. The bias arises due to past history of wins or loses.

Theorem V : The excitement in a biased event is directly proportional to sum of wins of individual participants expressed in percentage and directly proportional to unbiased excitement in the event.

Now consider the situation mathematically. Suppose there are ' $\mathrm{n}$ ' teams $\mathrm{N}_{1}, \mathrm{~N}_{2}, \mathrm{~N}_{3}, \ldots \ldots \ldots . . \mathrm{N}_{\mathrm{n}}$ and their percentage wins based on past history, respectively be $p_{1}, p_{2}, p_{3}, \ldots \ldots \ldots \ldots . p_{n}$.

Then,

The excitement in the biased event $\left(\mathrm{E}_{\mathrm{bi}}\right) \propto \frac{(\mathrm{p} 1+\mathrm{p} 2+\ldots+\mathrm{pn})}{100} \times \mathrm{E}_{\mathrm{i}}$

From the percentage of wins, $\mathrm{E}_{\mathrm{i}}$ can be obtained as :

$$
\mathrm{E}_{\mathrm{i}}=\sin \frac{\mathrm{p} 1 \pi}{(\mathrm{p} 1+\mathrm{p} 2+\ldots+\mathrm{pn})}+\sin \frac{\mathrm{p} 2 \pi}{(\mathrm{p} 1+\mathrm{p} 2+\ldots+\mathrm{pn})}+\ldots \ldots \ldots+\sin \frac{\mathrm{pn} \pi}{(\mathrm{p} 1+\mathrm{p} 2+\ldots+\mathrm{pn})}
$$

Therefore,

$$
\mathbf{E}_{\mathrm{bi}}=\mathrm{K}_{\mathrm{bi}} \frac{\mathbf{p} 1+\mathbf{p} 2+\cdots+\mathbf{p n}}{100} \times \mathrm{E}_{\mathrm{i}}
$$

Where ' $\mathrm{K}_{\mathrm{bi}}$ ' is a constant. ' $\mathrm{K}_{\mathrm{bi}}$ ' varies with observer as well as event. An observer investing more time or money in the event will have higher magnitude of ' $\mathrm{K}_{\mathrm{bi}}$ ' and dangerous games too will affect ' $\mathrm{K}_{\mathrm{bi}}$ ' magnitude.

But if we simply take a game and make the bias constant for all observers, by putting

$$
\mathrm{K}_{\mathrm{bi}}=1 \text { then, }
$$

$$
\mathbf{E}_{\mathrm{bi}}=\frac{\mathbf{p 1 + p 2 + \cdots + \mathbf { p n }}}{100} \times \mathbf{E}_{\mathbf{i}} \quad \text { (7) [the crowding effect equation] }
$$


Note :- For a non-supporter of two teams : say in a cricket match, an Indian will prefer watching England and Australia match instead of Bangladesh and Zimbabwe match.

For an observer (without extreme bias), supporting one of the participants teams ( say $\mathrm{N}_{1}$ ),

The excitement in the (biased) event is,

$$
\mathbf{E}_{\mathrm{bi1}}=\frac{\mathbf{p} \mathbf{1}}{(\mathbf{p} \mathbf{1}+\mathbf{p} \mathbf{2}+\ldots+\mathbf{p n})} \times \mathbf{E}_{\mathbf{i}}
$$

Thus for a biased observer supporting one of the teams, the excitement in the event is directly proportional to the probability of winning of that team.

\subsection{Extreme Bias Excitement}

For extreme bias, wherein the player or an observer has the only motive of winning by hook or crook; (we have to eliminate the probability tie), so let the probability of losing the match(failure) be 'p'.

Theorem VI : The excitement in the event for the extremely biased observer is directly proportional to the cosine of $\pi$ times the probability of failure.

Mathematically, it can be written as :

$\mathrm{E}_{\mathrm{bi} \mathrm{ix}} \propto \cos (\mathrm{p} \pi)$

$\mathrm{E}_{\mathrm{bi} \mathrm{ex}}=\mathrm{K}_{\mathrm{bi} \text { ex }} \cos (\mathrm{p} \pi)$

Where $\mathrm{K}_{\mathrm{bi} \text { ex }}$ is a constant which depends on the observer and ' $\mathrm{p}$ ' is the probability of failure.

Assuming $\mathrm{K}_{\mathrm{bi}} \mathrm{ex}=1$ for similar group of people and game,

$\mathbf{E}_{\text {bi ex }}=\cos (\mathbf{p} \pi) \longrightarrow(10)$

For values of $\mathrm{p}>0.5$, the excitement becomes negative, indicating depression. The depression will become maximum when the team, the observer is supporting has $100 \%$ chance of losing(failure).

There have been cases where people have died due to this negative excitement (depression) before the occurrence of the event.

4.2. Depression is a biased reaction to the event. Depression in a biased event is directly proportional to product of the probability of failure $\left(\mathrm{p}_{\mathrm{f}}\right)$ and excitement in the event.

Mathematically, Depression in a biased event $\left(\mathrm{D}_{\mathrm{bi}}\right)$,

$\mathrm{D}_{\mathrm{bi}} \propto \mathrm{p}_{\mathrm{f}} \times \mathrm{E}_{\mathrm{i}}$

$\mathrm{D}_{\mathrm{bi}}=\mathrm{K}_{\mathrm{di}} \times \mathrm{p}_{\mathrm{f}} \times \mathrm{E}_{\mathrm{i}} \quad$ [where $\mathrm{K}_{\mathrm{di}}$ is a constant]

Assuming $\mathrm{K}_{\mathrm{di}}=1$ for similar group of people and game

we get,

$\mathbf{D}_{\mathrm{bi}}=\mathbf{p}_{\mathrm{f}} \times \mathbf{E}_{\mathrm{i}}$

4.3. Excitement of Biased Event $\left(\mathbf{E}_{\mathbf{b o}}\right)$ : The excitement in the observer when the team he supports wins.

Theorem VII : For a biased observer/player (when the team wins), the excitement is directly proportional to the excitement in the event (biased) and inversely proportional to the probability of winning.

$E_{b o} \propto E_{b i} / p_{w}$, [where $E_{b i}$ is the excitement in the event, $p_{w}$ is the probability of winning].

$\mathrm{E}_{\mathrm{bo}}=\mathrm{K}_{\mathrm{bo}} \mathrm{E}_{\mathrm{bi}} / \mathrm{p}_{\mathrm{w}} \quad$ [where $\mathrm{K}_{\mathrm{bo}}$ is a constant]

Assuming $K_{\mathrm{bo}}=1$ for similar group of people and game,

$\mathbf{E}_{\mathrm{bo}}=\frac{\mathrm{Ebi}}{\mathbf{P w}}$

Note :- for a biased observer supporting a weak team, the excitement is greater when his team wins against the stronger team.

4.4. Depression of Biased Event $\left(\mathbf{D}_{\mathbf{b o}}\right)$ : It is the depression in the biased observer when the team he supports loses.

Theorem VIII : For a biased observer/player, when the team loses, the depression is directly proportional to the excitement in the biased event and inversely proportional to probability of losing(failure).

$\mathrm{D}_{\mathrm{bo}} \propto \mathrm{E}_{\mathrm{bi}} / \mathrm{p}_{\mathrm{f}} \quad\left[\mathrm{E}_{\mathrm{bi}}\right.$ is the excitement in the event, $\mathrm{p}_{\mathrm{f}}$ is the probability of failure]

$\mathrm{D}_{\mathrm{bo}}=\mathrm{K}_{\mathrm{do}} \mathrm{E}_{\mathrm{bi}} / \mathrm{p}_{\mathrm{f}}$ [where $\mathrm{K}_{\mathrm{do}}$ is a constant]

Assuming $\mathrm{K}_{\mathrm{do}}=1$ for similar group of people and game.

$\mathbf{D}_{\mathrm{bo}}=\mathbf{E}_{\mathrm{bi}} / \mathbf{p}_{\mathbf{f}}$

Note :- For a biased observer supporting a strong team, the depression is greater when his team loses against a weak team.

4.5. Excitement Impulse of Biased event (E.I..$_{\mathbf{b}}$ ) : The excitement generated in a biased observer after an event has occurred due to the un-expectancy of the event's occurrence. 
Theorem IX : The Excitement Impulse of a (biased) event is the product of excitement of the event (biased) and probability of not expecting the event's occurrence.

Mathematically,

E.I. $\mathrm{f}_{\mathrm{b}} \propto \mathrm{E}_{\mathrm{bo}} \times 1 / \mathrm{p}$, [where $\mathrm{E}_{\mathrm{bo}}$ is the excitement of event, $1 / \mathrm{p}$ is the probability of un-expectancy of event's occurrence]

E. $\mathrm{I}_{\mathrm{b}}=\mathrm{K}_{\mathrm{eio}} \mathrm{E}_{\mathrm{bo}} \times 1 / \mathrm{p}$ [where $\mathrm{K}_{\mathrm{eio}}$ is a constant]

Assuming $\mathrm{K}_{\mathrm{eio}}=1$ for similar group of people and game,

E.I. $\cdot \mathbf{b}=\mathbf{E}_{\mathrm{bo}} / \mathbf{p}$

\section{Economical Excitement (E.E.)}

Excitement arises when a person invests his money in the share market or business. The profit or loss directly affect the behaviour of the investor.

If in a work, ' $x$ ' be the amount invested by a person and ' $y$ ' be the amount that he receives (dividend) in all, for his investments, then :

Theorem X: The Economical Excitement for a person is proportional to the logarithmic ratio of amount received to the amount invested.

$\mathrm{E} . \mathrm{E} \propto \log (\mathrm{y} / \mathrm{x})$

$\mathrm{E} . \mathrm{E}=\mathrm{K}_{\mathrm{e}} \log (\mathrm{y} / \mathrm{x})$ [where $\mathrm{K}_{\mathrm{e}}$ is a constant]

Assuming $K_{e}=1$ for positive ' $y$ ' and ' $x$ ' values.

E.E. $=\log (\mathbf{y} / \mathbf{x})$

There may arise some special cases, which need evaluation :

Case $\mathbf{I}$ : when $\mathrm{y}=0$ for positive $\mathrm{x}$

The Excitement cannot be defined as such. So, we introduce an arbitrary constant 'c' into the logarithmic equation, so that the multiples may not change with change in currency.

Therefore,

E.E. $=\log \left(\frac{c+y}{x}\right)$, but as $y=0$

So, $\quad$ E.E. $=\log \mathrm{c}-\log \mathrm{x}$

If we assume $\mathrm{c}=1$ for similar group of people and investment, then we get

E.E. $=\log 1-\log \mathrm{x}=0-\log \mathrm{x}$

E.E. $=-\log x$

Therefore when the return from an investment is zero, the excitement is directly proportional to the logarithm of the amount invested. The negative sign shows the negative excitement, that is depression.

Case II : when y $<0$

In this case, the person has incurred a loss. So the overall value will be added to the amount invested.

E.E. $=\log \left(\frac{c}{x+y}\right)=\log c-\log (x+y)$

Assuming, $\mathrm{c}=1$

Therefore, $\quad$ E.E. $=-\log (x+y)$

Thus in this case there is negative excitement which is proportional to the logarithm of sum of amount invested and debt incurred.

\section{Applications on Population Groups}

The excitement in human nature have led to problems in population risk groups which include deaths due to suicides and heart attacks. Excitement may also lead to commission of crimes such as homicides, gambling/betting and match-fixing.

\subsection{Suicide}

The act of killing one self is suicide. Those who commit suicide are either in stress or mentally ill. We shall consider the former in this paper, as it turns out to be an increasing health problem of the modern society. Failure is the chief cause of stress which leads to depression and impulsivity to commit suicide. 


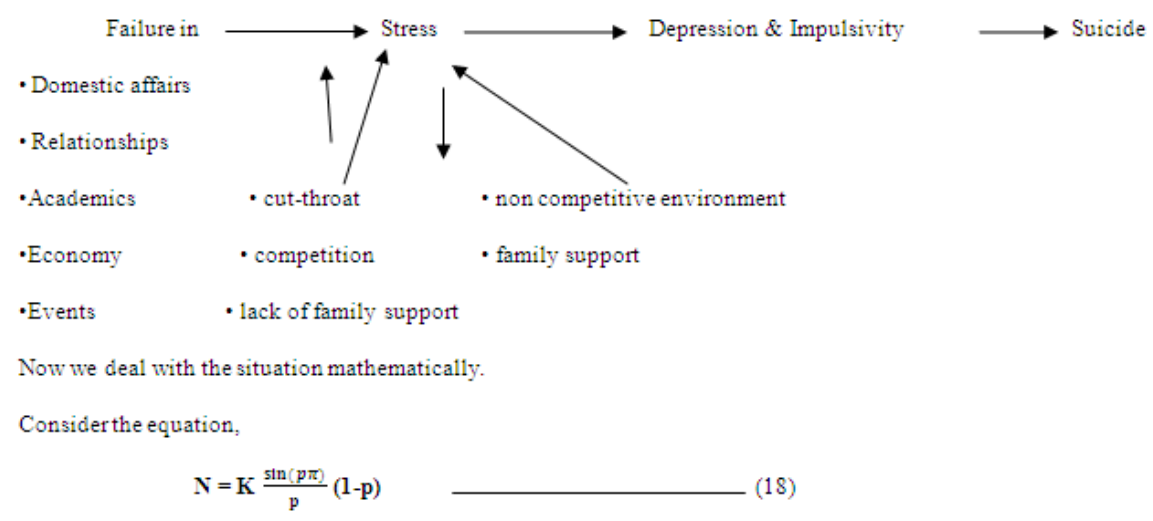

Where $\mathrm{N}$ is number of deaths due to suicide, 'p' is the probability of failure, $\mathrm{K}$ is a constant for a risk group.

From the equation it can be observed that after an event's occurrence, the number of suicides (or probability of committing suicide) $\mathrm{N}$, is directly proportional to the un-expectancy of failure. Thus a person who expects least of failures, when fails will have greater chance of committing suicide due to disappointment and depression than someone who expects and is accustomed to failure.

In the above equation (18),

When, $\mathrm{p}=0.5$

Then, $\quad \mathrm{N}=\mathrm{K}$

Thus, $\mathrm{K}$ is numerically equal to probability (or number) of deaths when there is $50 \%$ chance of failure. Therefore in an examination, a student of having $75 \%$ chance of success will have 2.1 times more chance of committing suicide than a student having $50 \%$ chance of success, given that both of them fails.

Taking another example, consider a group having equal income investing in a company. The number of deaths in people investing $90 \%$ of their income will be 12 times more than those who invested $25 \%$ of their income, if the company goes bankrupt. So that gives the concept of not putting all the eggs in one basket while making investments.

Now consider another situation. The number of students appearing in a competitive entrance examination is say, 6 lakhs. There are only 50 thousand seats. So the probability of failure associated with each of them is very high. There is extreme bias because every individual wants to win (get through the competitive exams). If suppose there are 350 deaths after the results have been declared, what could be the possible number of deaths when next year 7 lakhs students appear in the competitive exam?

The bias is extreme and the study of population shows that death will be proportional to the probability of failure. The equation for number of deaths turns out to be a combination of equations (10) and (11) where $E_{i}$ is replaced by $\mathrm{E}_{\mathrm{bi} \text { ex }}$

Therefore,

Number of deaths $\quad \mathrm{N}=\mathrm{K} \mathrm{p}_{\mathrm{f}} \mathrm{x}|\cos (\mathrm{p} \pi)|$

In first case, 5.5 lakhs students are under risk. The probability of failure is $5.5 / 6$ and number of deaths is $350 / 5.5$ per lakhs population.

In second case, 6.5 lakhs students are under risk. The probability of failure is $6.5 / 7$, so using the above equation, it can be written as,

$\mathrm{N}_{1} / \mathrm{N}_{2}=\mathrm{p}_{1}\left|\cos \left(\mathrm{p}_{1} \pi\right)\right| / \mathrm{p}_{2}\left|\cos \left(\mathrm{p}_{2} \pi\right)\right|$

$\mathrm{N}_{2}=\mathrm{N}_{1} \mathrm{p}_{2}\left|\cos \left(\mathrm{p}_{2} \pi\right)\right| / \mathrm{p}_{1}\left|\cos \left(\mathrm{p}_{1} \pi\right)\right|$

$\mathrm{N}_{2}=350 / 5.5 \times 6.5 / 7 \times|\cos (6.5 / 7 \pi)| / 5.5 / 6 \times|\cos (5.5 / 6 \pi)|=65$ per lakh

The risk group is 6.5 lakhs population.

So the number of deaths ought to be 423 . Thus the death rate increases with more number of students appearing in the examination. It will increase due to more number of attempts due to failure leading to depression. The peer pressure, family pressure and the social environment all acts on the individual to evolve a complex human behaviour and so deviations may occur. But the basic equation for a do or die situation will remain same, and like Einstein said, that the most complex laws can be reduced to simple equations; so can the human behaviour be.

When events are biased and probability of failure is not high, then number of self harm attempts can be derived from the equation as :

$\mathrm{N}=\mathrm{KE}_{\mathrm{bo}} / \mathrm{p}$ [where $\mathrm{p}$ is the probability of failure] 
Thus it can be observed with time, the bias in human nature is increasing and more deaths are likely to occur. Where earlier, death due to failure had inverse relationship to probability of failure; due to bias, death will be inversely proportional to the square of probability of failure.

Suicide is therefore, a major public health problem in the modern society and unfortunately it is still ignored. Suicide rate has turned out to be an index of development, the developed countries having higher suicide rates. With the conquering of infectious disease, the excitement factor is going to play a major role in causation of deaths in the urban population or in the developed countries. Our country is developing and deaths due to suicide and cardio-vascular disease is increasing.

\subsection{Deaths due to heart failure}

It is a matter of common experience that a news of grave consequences or an exciting news when revealed causes marked physiological changes in the body. For this reason exciting or depressing news are withheld and gradually revealed to an elderly or someone suffering from a disease that will likely affect the health of the person.

(i). Using this analogy in games; when matches are played, and a large number of observers are watching the match, then deaths are more likely to occur due to cardio-vascular failure, when unexpected events occur. However in a game, it has been observed that there are more number of deaths if the excitement is maintained high till the end of the match. It will be maximum when climax occurs at the end of the time interval of the game.

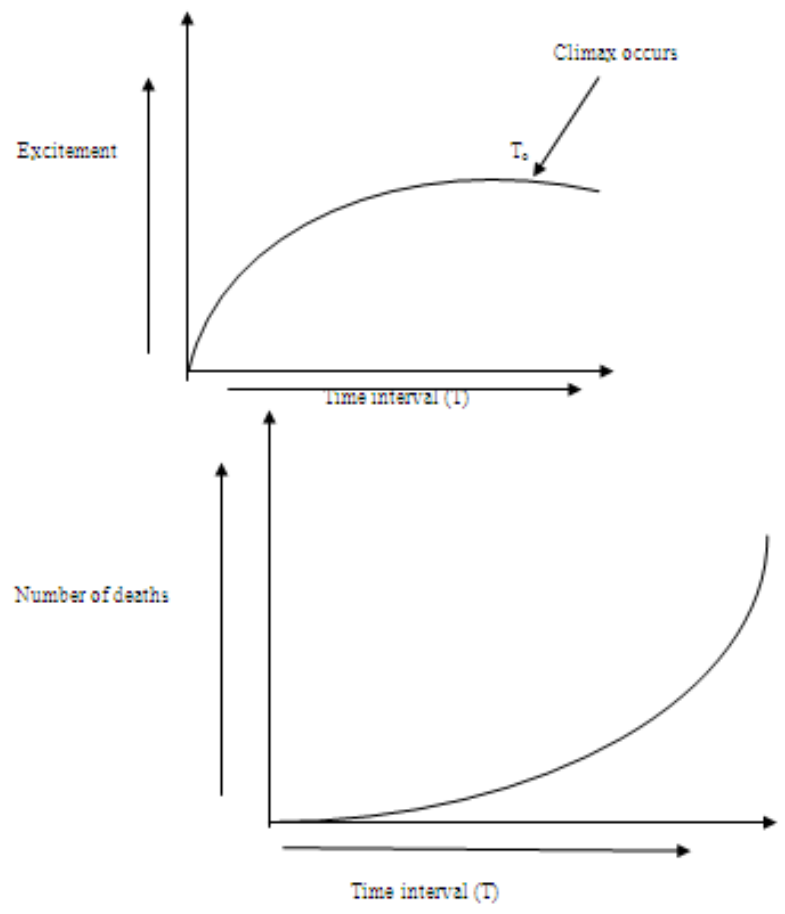

If the excitement is maintained high as such, the number of deaths will follow a curve as shown in the graph. We can use the following equation to determine the number of deaths.

$\mathrm{N}=\mathrm{K}\left(\mathrm{T}_{\mathrm{o}} / \mathrm{T}\right)^{2} \frac{\sin (\mathrm{p} \pi)}{\mathrm{p}}[1-\mathrm{p}-\mathrm{p}(\mathrm{tie})]$

Where $T_{o}$ is the time interval when the climax occurs and ' $T$ ' is the total time interval of the game.

There are usually no deaths when a match ends in a draw. Matches in which an observer supports a team will more likely to cause death if the team wins or loses at the last moment.

(ii). Death may also occur due to economical excitements. This is usually seen when a large profit or dividend is obtained from low investment. The best examples are, when a person wins a lottery or finds a bag of money or hidden treasures.

$$
\begin{aligned}
& \text { Using equation } \\
& \text { E.E. }=\log (y / x) \text {, for } y>x
\end{aligned}
$$

The 'Economical Excitement' in the event is extremely high and emotional changes may cause heart failure. People used to seeing less money are more prone to the excitement. 50 years earlier, when less money was in circulation and life wasn't as fast as today, the economical excitement wasn't as high as present day. So there were less number of deaths. But then with increase in population and capital growth, more deaths are likely to occur due to economical excitement. And one of the consequences of capital growth that has lead to 
increased economical excitement is the fast life style of the present generation. But again, people used to seeing large capital will get used to the excitement.

\subsection{Homicidal death}

High excitement may also lead to the commission of homicides. The excitement of the event can be calculated and the result applied to see if the death occurred in warm blood or cold blood. It could be of use to forensic experts. When the excitement of the event is high, the probability of death due to warm blood increases.

\subsection{Betting/Gambling and Match-fixing}

The excitement in games have led to betting. Matches that have high excitement in the event $\left(\mathrm{E}_{\mathrm{i}}\right)$ are watched by a large number of people. So with increasing excitement, and the number of people observing the match, the probability and number of betting increases.

Now, when the chances of one team winning is more than the other, and a large number of people have bet in favour of the team that has greater probability of winning; then if that team loses, then those in support will lose the bet. And those gambling uses this situation to their advantage. When a team having less chances of winning, wins (less number of people have laid bet on it), the excitement of the event is high. Those gambling will more reliably fix the match in these situations to their advantage. Therefore, the probability of a match being fixed increases with increase in excitement of the event $\left(\mathrm{E}_{\mathrm{o}}\right)$, and is proportional to it.

\section{Conclusion}

As a doctor I observed some patients coming to the hospital after cricket match. Most of them happened to have heart attacks when team India lost or one of their favourite players played badly.

This theory generalizes not only cricket, but all games and events of contest in this world. It expresses the emotion in mathematical terms both for a player and observer. Surely there is some deep connection between the events and human behaviour. And this could be explained in terms of excitement which needs a suitable mathematical correlation. And though it may appear strange, as it was when Gregor Mendel proposed his theory, but a second thought will reflect that all complex biological behaviours do indeed have simple mathematical base which can be expressed in equation form. And all it needs is a suitable analogy.

These equations can be applied on the related population groups and thus human behaviour may reliably be estimated and predicted. The further use of this theory could be in understanding the basics of human emotions, in economics and in building of androids with human emotions. The usage in economics is tremendous, as this theory can be applied to determine that events having high excitement in event will be watched by a huge crowds and events having high excitement of event will make news. This theory is just a little stepping stone in the realm of a much larger complex mathematical human emotion chapter.

\section{References}

